Supplement of Biogeosciences, 14, 1039-1054, 2017

http://www.biogeosciences.net/14/1039/2017/

doi:10.5194/bg-14-1039-2017-supplement

(C) Author(s) 2017. CC Attribution 3.0 License.

(c) (i)

Supplement of

\title{
Carbon balance of a grazed savanna grassland ecosystem in South Africa
}

Matti Räsänen et al.

Correspondence to: Matti Räsänen (matti.rasanen@ helsinki.fi)

The copyright of individual parts of the supplement might differ from the CC-BY 3.0 licence. 


\section{Vegetation sampling}

The vegetation surrounding the measurement station was classified based on land-use and vegetation structure (Figure S1), for which a detailed sampling was performed four times during the period from April 2011 to January 2012. Seven homogeneous land-use units were identified, including maize fields, dry sandy grassland, moist sandy grassland, disturbed

5 grassland, thornveld savannah, woodland savannah and plantation. Grasslands had a tree cover of less than 15\%, whereas in savannas it was greater than $15 \%$. Furthermore, savannas were divided into thornveld, which had sparsely distributed large trees, and woodland with a tree cover greater than $50 \%$.

The central region of each homogeneous unit was sampled along a transect, which resulted in 42 plots in total, six per transect. Each of these plots had an area of $100 \mathrm{~m}^{2}$. All of the plant species were counted and identified up to species level,

10 and their major growth form was recorded. In addition, for all the woody species higher than $1 \mathrm{~m}$, the mean height, mean canopy height and width of each species were determined.

Within each plot, leaf material was collected from four $1 \mathrm{~m}^{2}$ subplots during each of the four periods, and a one-sided leaf area index (LAI) of all the leaves was determined for grasses, forbs, shrubs and trees. After this all the plant material was dried for three days and weighed.

15 


\section{Tables}

Table S1: Soil structural and chemical composition of each homogeneous land-use unit. MF, Maize fields; WL, Woodland; TV, Thornveld; P, Plantation; DSG, Dry sandy grassland, MSG, Moist sandy grassland, DG, Disturbed grassland.

\begin{tabular}{|c|c|c|c|c|c|c|}
\hline & MF & WL & TV \& P & DSG & MSG & $\overline{D G}$ \\
\hline Medium-Coarse sand & 43.1 & 49.1 & 45.1 & 47.2 & 37.9 & 44.1 \\
\hline Fine sand & 44.0 & 37.8 & 40.1 & 37.7 & 41.3 & 45.6 \\
\hline Silt & 3.5 & 7.6 & 7.8 & 8.0 & 8.5 & 3.4 \\
\hline Clay & 9.4 & 4.7 & 7.0 & 7.2 & 12.3 & 7.0 \\
\hline Org. cont. (\%) & $3-6$ & $3-6$ & $3-6$ & $3-6$ & $3-6$ & $3-6$ \\
\hline Organic-C (\%) & 0.68 & 0.87 & 0.93 & 0.91 & 1.01 & 0.54 \\
\hline Acidity $(\mathrm{pH})$ & 5.12 & 5.73 & 5.69 & 5.09 & 6.52 & 5.22 \\
\hline CEC $(\mathrm{cmol}(+) / \mathrm{kg})$ & 9.56 & 11.63 & 12.1 & 11.37 & 12.42 & 10.42 \\
\hline S-value $(\operatorname{cmol}(+) / \mathrm{kg})$ & 2.63 & 4 & 3.42 & 2.29 & 9.51 & 1.05 \\
\hline Base saturation (\%) & 27.52 & 34.38 & 28.27 & 20.12 & 76.62 & 10.05 \\
\hline Nitrogen $(\%)$ & 0.01 & 0.06 & 0.07 & 0.07 & 0.08 & 0.03 \\
\hline Calcium (mg/l) & 373 & 605 & 449 & 267 & 1009 & 108 \\
\hline Magnesium (mg/ l) & 74 & 97 & 101 & 69 & 477 & 37 \\
\hline Potassium (mg/l) & 337 & 230 & 349 & 365 & 583 & 253 \\
\hline Phosphorus (mg/l) & 3 & 3.54 & 3.31 & 2.89 & 3.42 & 2.78 \\
\hline Sodium (mg/l) & 0.5 & 7.5 & 4.5 & 6.5 & 26 & 5.5 \\
\hline Sulphur (mg/l) & 16.4 & 13.88 & 14.39 & 12.95 & 13.16 & 11.54 \\
\hline Arsenic (mg/kg) & 1.074 & 1.06 & 1.213 & 1.436 & 1.402 & 1.2 \\
\hline Aluminium (mg/kg) & 8205 & 4655 & 5993 & 6705 & 9517 & 5963 \\
\hline Cadmium (mg/kg) & 0.016 & 0.026 & 0.022 & 0.026 & 0.028 & 0.017 \\
\hline Chromium(mg/kg) & 163 & 48 & 110 & 84 & 111 & 86 \\
\hline Cobalt (mg/kg) & 10.26 & 5.32 & 8.97 & 4.29 & 10.1 & 3.35 \\
\hline Copper (mg/kg) & 11.83 & 7.97 & 9.85 & 9.3 & 13.03 & 7.16 \\
\hline Iron $(\mathrm{mg} / \mathrm{kg})$ & 16365 & 9965 & 14778 & 12448 & 17313 & 11168 \\
\hline Lead (mg/kg) & 3.28 & 3.34 & 3.51 & 3.86 & 5.3 & 3.1 \\
\hline Manganese $(\mathrm{mg} / \mathrm{kg})$ & 465 & 350 & 395 & 218 & 625 & 157 \\
\hline Nickel (mg/kg) & 38 & 11 & 29 & 18 & 30 & 10 \\
\hline Zinc $(\mathrm{mg} / \mathrm{kg})$ & 9.9 & 8.32 & 7.36 & 9.57 & 11.15 & 6.21 \\
\hline
\end{tabular}




\section{Figures}

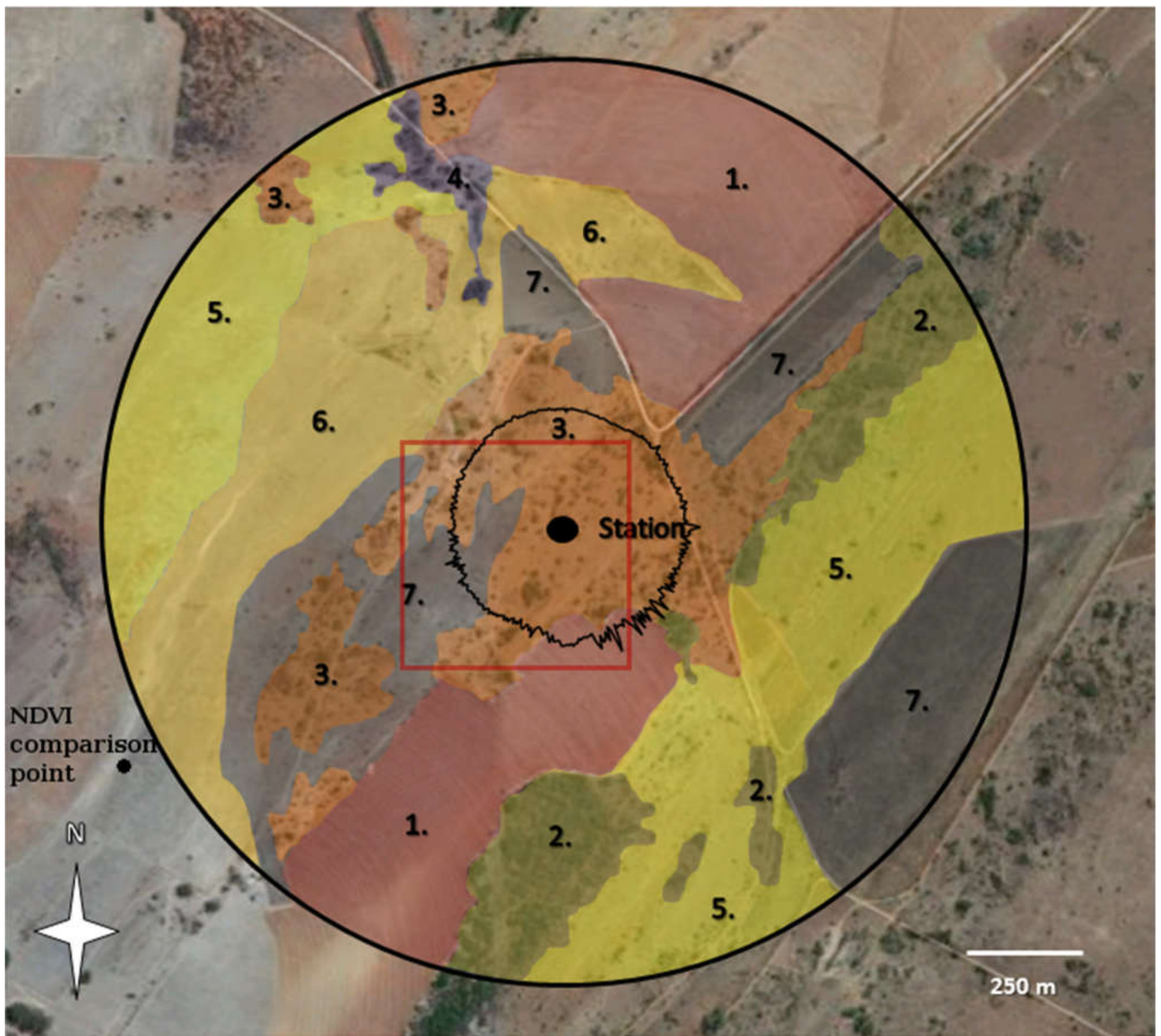

\section{Maize fields 3. Thornveld 5. Dry sandy grassland 7. Disturbed grassland 2. Woodland 4. Plantation 6. Moist sandy grassland}

40 Figure S1. Satellite image of the Welgegund measurement site showing the seven different sampling regions based on different vegetation and land-use classes. The red square shows a MODIS NDVI pixel at $500 \mathrm{~m}$ spatial resolution. The black contour indicates mean $80 \%$ cumulative flux footprint. The black dot on the bottom left corner indicates the moist sandy grassland area which was used in the NDVI comparison. 


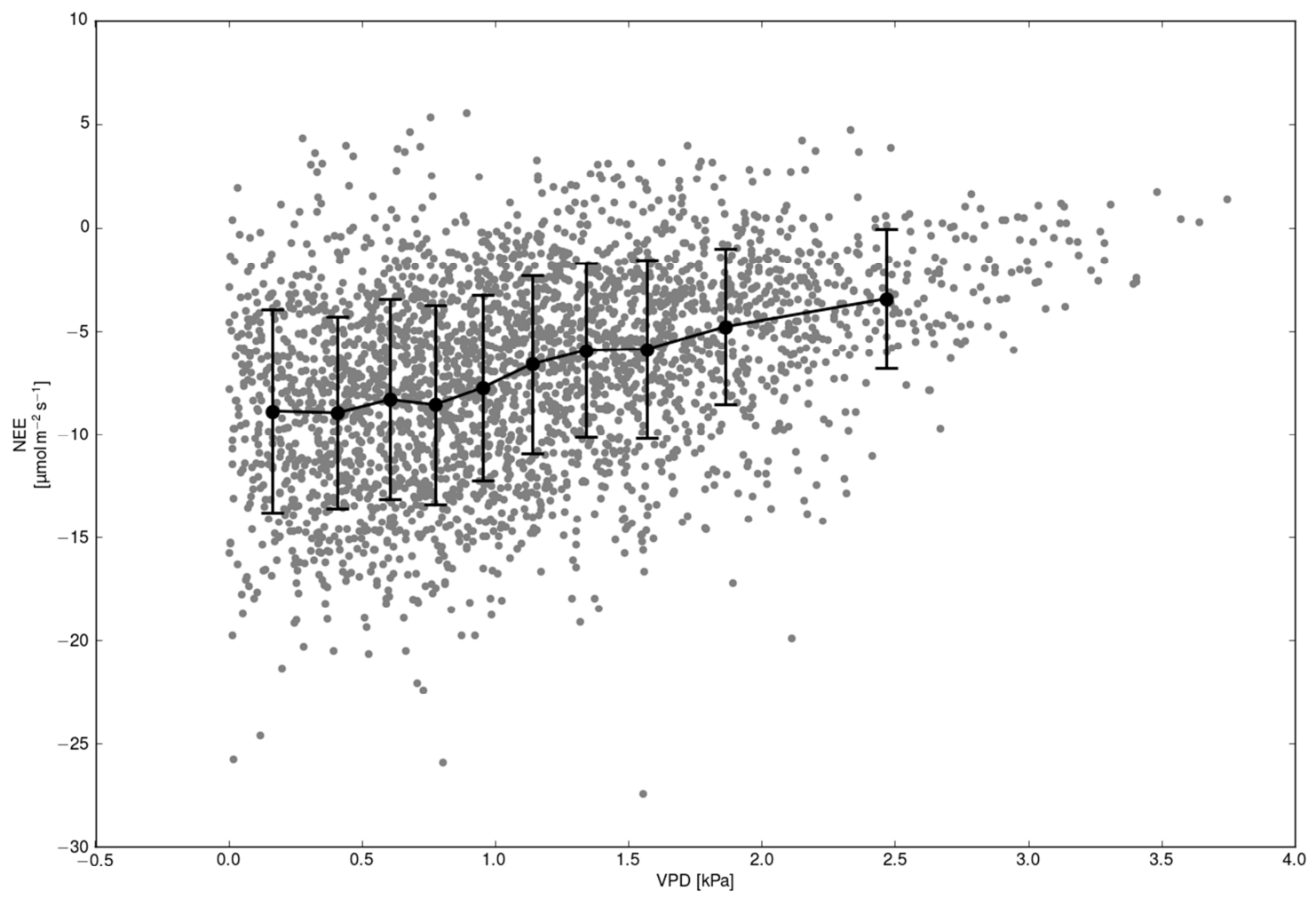

Figure S2. Relationship between VPD and daytime NEE (Net radiation $>500 \mathrm{~W} \mathrm{~m}^{-2}$ ) between $1^{\text {st }}$ of September 2010 and $31^{\text {st }}$ of 50 August 2013. Each bin contained 290 values. 


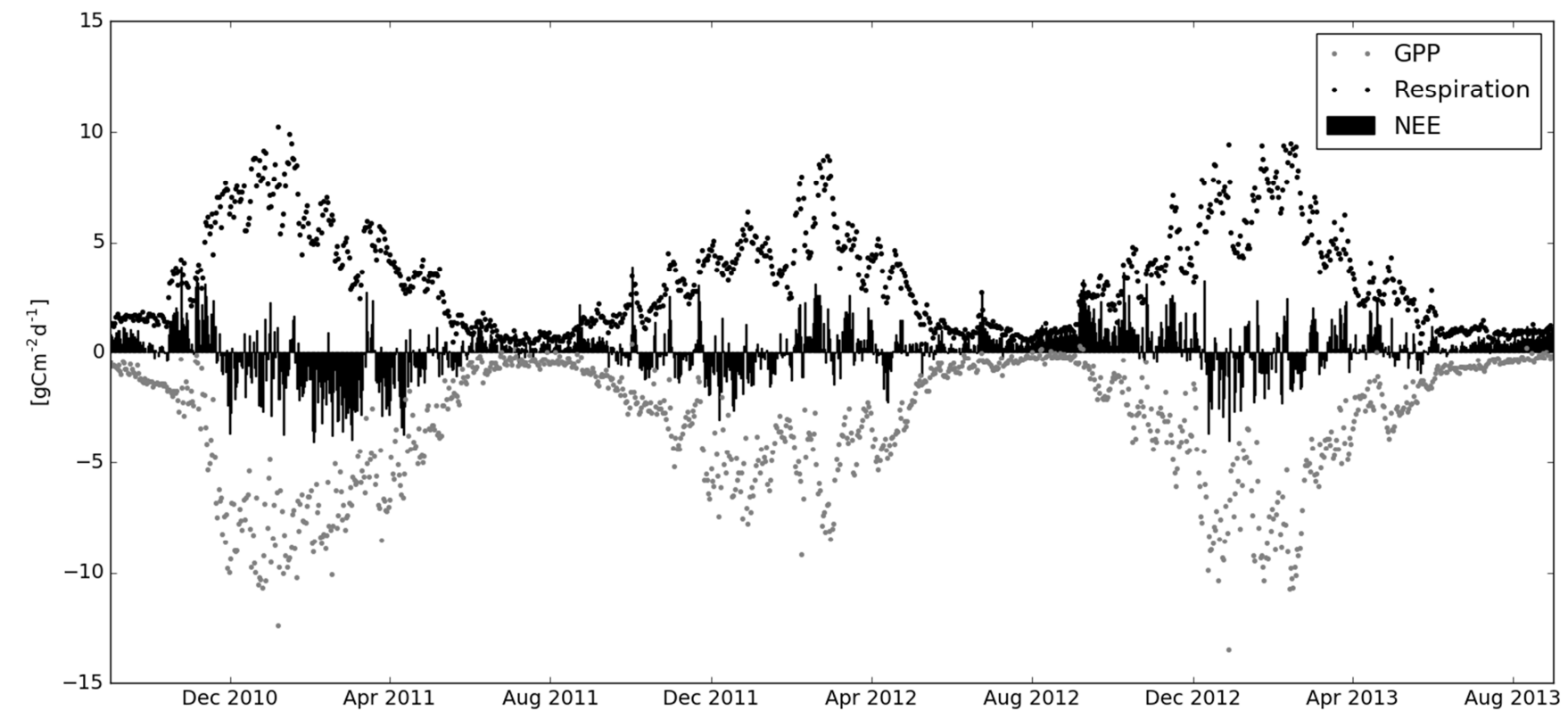

Figure S3. Daily sum of NEE, GPP and respiration for the whole measurement period. 